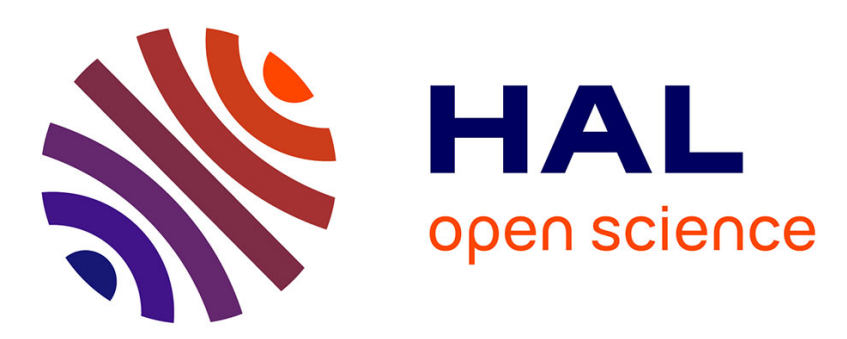

\title{
Traitement de signal ultrasonore adapté à l'évaluation non destructive de matériaux dispersifs
}

\author{
Bertrand Audoin, J. Roux
}

\section{To cite this version:}

Bertrand Audoin, J. Roux. Traitement de signal ultrasonore adapté à l'évaluation non destructive de matériaux dispersifs. Revue de Physique Appliquée, 1990, 25 (10), pp.1011-1017. 10.1051/rphysap:0199000250100101100 . jpa-00246267

\section{HAL Id: jpa-00246267 https://hal.science/jpa-00246267}

Submitted on 1 Jan 1990

HAL is a multi-disciplinary open access archive for the deposit and dissemination of scientific research documents, whether they are published or not. The documents may come from teaching and research institutions in France or abroad, or from public or private research centers.
L'archive ouverte pluridisciplinaire HAL, est destinée au dépôt et à la diffusion de documents scientifiques de niveau recherche, publiés ou non, émanant des établissements d'enseignement et de recherche français ou étrangers, des laboratoires publics ou privés. 
Classification

Physics Abstracts

$43.35 \mathrm{C}-46.30 \mathrm{C}-62.30 \mathrm{D}$

\title{
Traitement de signal ultrasonore adapté à l'évaluation non destructive de matériaux dispersifs
}

\author{
B. Audoin et J. Roux \\ Laboratoire de Mécanique Physique, U.R.A. C.N.R.S. n 867 , Université de Bordeaux I, 351 Cours de la \\ Libération, 33405 Talence Cedex, France
}

(Reçu le 14 mai 1990, accepté le 5 juillet 1990)

\begin{abstract}
Résumé. - La caractérisation mécanique des milieux anisotropes est souvent rendue difficile par une forte dispersion et le chevauchement des impulsions ultrasonores propagées. Nous proposons une mesure des temps de vol basée sur la transformée de Hilbert de la fonction de transfert de propagation. Par comparaison avec l'intercorrélation des signaux d'entrée et de sortie, elle offre une meilleure résolution des chevauchements et permet un calcul de la vitesse asymptotique à basse fréquence peu sensible à la dispersion.

Abstract. - In the non destructive evaluation of many anisotropic media, difficulties arise in conjunction with a coarse dispersion and echoes overlapping. We propose a method based on the Hilbert transform of the propagation transfer function. In comparison with the classical input-output intercorrelation, Hilbert method offers a better resolution in overlapping and leads to the asymptotic low frequency value of the velocity with a low dependence of dispersion.
\end{abstract}

\section{Introduction.}

La caractérisation mécanique des matériaux anisotropes par ultrasons consiste à retrouver la matrice des raideurs réelles ou complexes du milieu, à partir de mesures de vitesses de propagation d'ondes planes dans un ensemble de directions bien choisies [1]. Dans les méthodes impulsionnelles, la vitesse est obtenue en divisant le chemin parcouru par le « temps de vol » d'une impulsion. Cette mesure peut être rendue délicate par les conditions de propagation. Beaucoup de matériaux composites artificiels ou naturels de par leur hétérogénéité et les dimensions disponibles d'échantillons peuvent rendre les techniques peu précises voire inopérantes. Le chevauchement d'échos multiples par réflexions internes ou mélange de modes à vitesses voisines ne permettent plus d'isoler correctement la réponse d'espace libre. Il suffirait donc d'utiliser des impulsions plus courtes; la dispersion devient alors prohibitive, l'effet de filtre passe-bas étale les réponses temporelles tandis qu'apparaissent d'autres effets liés à des longueurs d'ondes trop courtes par rapport aux échelles structurales du milieu. Dans de telles conditions, les algorithmes d'optimisation des constantes élastiques peuvent converger vers des minima fictifs et donner des valeurs numériques dénuées de réalité $[2,3]$.

\section{La lame à faces parallèles immergée.}

Cette technique expérimentale abondamment utilisée et décrite $[4,5]$, illustre bien les difficultés majeures rencontrées. La bonne reproductibilité des interfaces, la possibilité d'obtention de tous les coefficients élastiques à partir d'un seul échantillon, rapidité et faible coût sont ses atouts majeurs. Ses limitations sont essentiellement liées à des considérations dimensionnelles. Le choix crucial est celui de la fréquence centrale des transducteurs vis-à-vis des longueurs caractéristiques.

L'étendue du domaine spectral est limitée vers le bas par l'épaisseur de la lame responsable d'un chevauchement trop serré des signaux et vers le haut par une absorption trop grande et la violation des hypothèses d'homogénéité en regard des échelles structurales du milieu. Ce domaine utilisable peut être très étroit. Le succès de la méthode repose alors sur l'aptitude des traitements de signaux à la résolu- 
tion d'échos chevauchants en présence d'une dispersion non négligeable. Le temps de vol estimé pour chacun d'eux doit pouvoir être relié sans ambiguité aux caractéristiques mécaniques du milieu.

L'exemple de la figure 1 est celui d'une impulsion de fréquence centrale $1 \mathrm{MHz}$ ayant transité sous incidence oblique dans une lame d'épaisseur trop faible en regard de la différence des vitesses des deux modes générés par réfraction. Il est clair que dans ce cas les méthodes habituelles de chronométrie de niveau ou de superposition d'échos (pulse overlap) sont inopérantes. Seules les techniques de déconvolution ou de filtrage optimal peuvent donner des résultats exploitables.

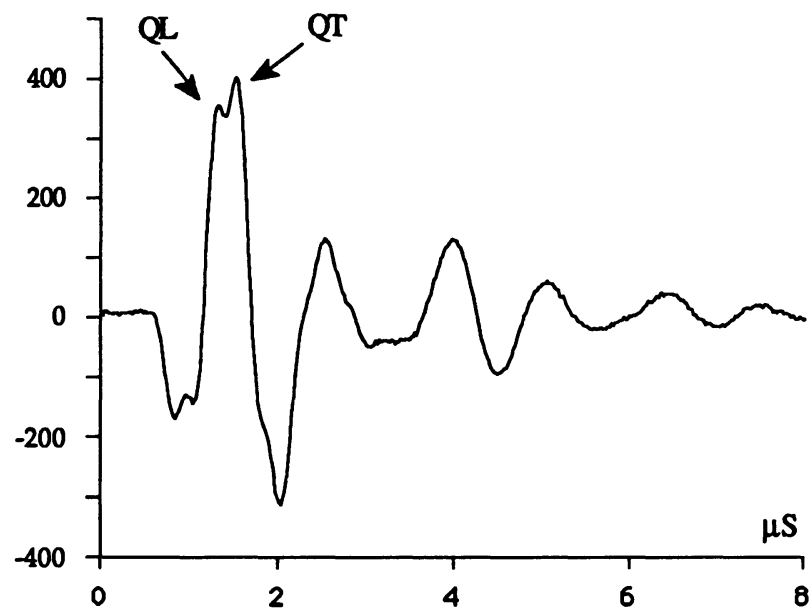

Fig. 1. - Chevauchement des modes quasi-longitudinal (QL) et quasi-transversal (QT) à la sortie d'une lame de $3,5 \mathrm{~mm}$ d'épaisseur en composite carbone-époxy unidirectionnel sous une incidence de $7,5^{\circ}$ dans l'eau.

[Overlapping of QL and QT modes through a one axis carbone-epoxy sample of $3.5 \mathrm{~mm}$ thickness at an incidence angle of $7.5^{\circ}$ in water.]

\section{Le filtre propagatif.}

Le transfert total, réfraction comprise, au travers de la lame s'écrit simplement par une équation de convolution (*) entre le signal d'entrée $e(t)$ obtenu en l'absence d'échantillon et le signal $s(t)$ recueilli au travers de celui-ci :

$$
s(t)=e(t) * h(t) \leftrightarrow S(f)=E(f) . H(f)
$$

où $H(f)$ est analytique et hermitienne car transformée de Fourier d'une fonction causale réelle. On sait que pour une propagation par ondes planes de parcours $x$, elle s'écrit simplement :

$$
H(f)=\exp [-\alpha(f) x] \cdot \exp [-i k(f) x]
$$

expression dans laquelle l'atténuation $\alpha(f)$ et le nombre d'onde $k(f)$ sont reliés par les relations de Kramers-Krönig exprimant la causalité de $h(t)$.
Les propriétés de parité de $\alpha(f)$ et d'imparité de $k(f)$ permettent d'écrire ce dernier sous la forme :

$$
k(f)=2 \pi f . \ell(f), \text { où }
$$

où

$$
\ell(f)=1 / C(f) \text { est la « lenteur » de phase . }
$$

Compte tenu de sa parité elle peut s'écrire sous la forme d'un développement convergent en série entière de puissances paires de la fréquence :

$$
\ell(f)=\ell(0)+\phi(f), \quad \phi(f)=\sum_{1}^{\infty} \ell_{2 k} f^{2 k}
$$

la fonction de transfert définie en (2) prend alors la forme :

$$
\begin{aligned}
& H(f)=G(f) \cdot \exp [-i 2 \pi f \tau] \\
& G(f)=\exp [-\alpha(f) x] \cdot \exp [-i 2 \pi f x \phi(f)]
\end{aligned}
$$

où $\tau=x . \ell(0)=x / C(0)$ est le retard de propagation asymptotique à basse fréquence directement lié aux constantes élastiques statiques du milieu. Son identification précise en présence de dispersion et d'échos multiples est l'objectif majeur de ce travail. Pour la bonne compréhension des raisonnements qui vont suivre il est important de remarquer que, par transformation de Fourier inverse de (5) on obtient simplement :

$$
\begin{aligned}
h(t)=g(t) & * \delta(t-\tau)= \\
& =g(t-\tau) \leftrightarrow g(t)=h(t+\tau)
\end{aligned}
$$

où $g(t)$, dont la transformée de Fourier est définie par (5) et (4), n'est autre que $h(t)$ elle-même mais translatée du seul retard à fréquence basse que l'on veut mesurer. Elle contient évidemment toutes les informations relatives à la dispersion du milieu. Sa modélisation requiert des hypothèses supplémentaires sur les mécanismes dissipatifs mis en jeu (absorption, diffraction, diffusion, relaxation...) que nous n'aborderons pas ici.

\section{Intercorrélation entrée-sortie.}

Déjà décrite et discutée en l'absence de dispersion [6] on peut montrer qu'elle n'est autre qu'une identification de filtre optimal au sens des moindres carrés. Reprenons la notation de la relation (1) où les données acquises sont $e(t)$ et $s(t)$, le filtre à identifier $h(t)$.

$y(t)=g(t) * \delta(t-\tau) * e(t)=g(t) * e(t-\tau)$

est la réponse théorique supposée après transfert par le filtre. Au sens des moindres carrés, la fonction :

$$
F(\tau)=\int_{R}[s(t)-g(t) * e(t-\tau)]^{2} \mathrm{~d} t
$$


sera minimale pour $\tau=x . \ell(0)$ valeur optimale du retard. Par dérivation il vient :

$$
\begin{aligned}
\mathrm{d} F / \mathrm{d} \tau=2 \int_{R}[s(t)- & g(t) * e(t-\tau)] \times \\
& \times g(t) * e^{\prime}(t-\tau) \mathrm{d} t=0
\end{aligned}
$$

qui peut s'écrire, compte tenu de (6) :

$$
\begin{aligned}
\int_{R} s(t) \cdot y^{\prime}(t-\tau) \mathrm{d} t & \\
& =\int_{R} y(t-\tau) \cdot y^{\prime}(t-\tau) \mathrm{d} t=0
\end{aligned}
$$

dont le deuxième membre est une intégrale immédiate qui vaut $y^{2}(+\infty)-y^{2}(-\infty)=0$ car $y$ est une fonction causale donc à support borné.

Le premier membre de (9) n'est autre que la dérivée de la fonction d'intercorrélation $C(s / y)$ du signal de sortie $s(t)$ par le signal d'entrée $e(t)$ luimême convolué par la réponse impulsionnelle $h(t)$ inconnue a priori.

Si la dispersion est négligeable, $g(t)=\delta(t)$. L'équation (9) montre que la dérivée de l'intercorrélation entrée-sortie s'annule pour la valeur optimale du retard. Le zéro de la dérivée correspond alors à un maximum de la fonction $C(s / e)$.

Lorsque la dispersion est importante, l'extrémum de $C(s / e)$ ne coïncide plus avec celui de $C(s / y)=$ $C(s / e * g)$. Le temps de vol ainsi mesuré n'est plus la valeur recherchée $x . \ell(0)$, figure 8 .

D'autre part, le filtrage passe-bas par $g(t)$ accroît le chevauchement des échos multiples, rendant leur séparation moins précise, d'autant que pour les signaux ultrasonores usuels la fonctionnelle (7) n'est pas strictement convexe. L'algorithme de recherche d'extrémum peut alors converger vers un maximum relatif erroné.

Tant que l'hypothèse du retard pur peut être retenue, la caractérisation mécanique par spectrointerférométrie et corrélation donne donc de bons résultats. A titre d'illustration nous montrons sur la figure 2 les courbes de lenteurs normalisées (inverse des vitesses rapportées à celle de l'eau et représentées en coordonnées polaires en fonction de l'angle réfracté) obtenues sur un échantillon de carboneépoxy unidirectionnel d'épaisseur $3,5 \mathrm{~mm}$, en utilisant des transducteurs de fréquence centrale $5 \mathrm{MHz}$ dont la réponse impulsionnelle est suffisamment courte pour que les modes longitudinal et transversal soient temporellement bien séparés alors que leurs vitesses sont voisines à l'incidence de $7,5^{\circ}$. Sur cette figure 2, les courbes continues sont tracées à partir des valeurs expérimentales par une méthode d'optimisation [7].

Pour le même échantillon, mais avec des transducteurs de fréquence centrale $1 \mathrm{MHz}$, les signaux de sortie ont l'allure de celui de la figure 1 . Le chevau-

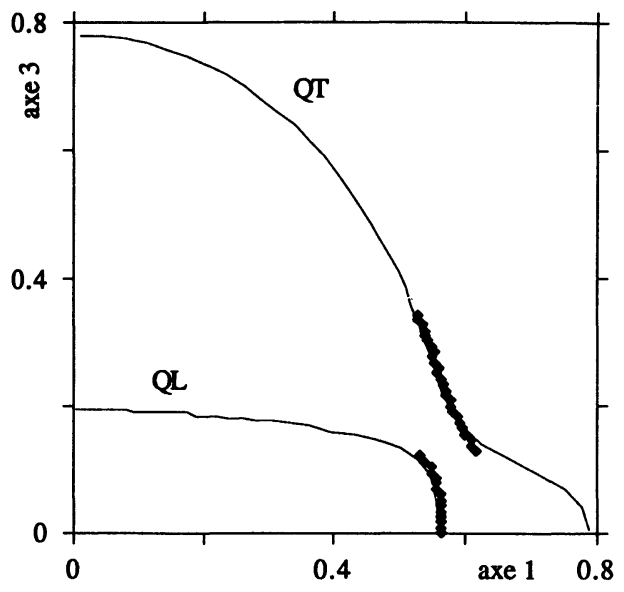

Fig. 2. - Valeurs expérimentales à $5 \mathrm{MHz}$ et courbes de lenteur calculées par intercorrélation entrée-sortie.

[Experimental points and optimal slowness curves at $5 \mathrm{MHz}$ computed by input-output intercorrelation.]

chement est tel que la différenciation des modes par intercorrélation n'est plus possible. La zone encadrée de la figure 3 illustre l'inefficacité de la méthode dans ce cas. Avec de telles valeurs expérimentales,

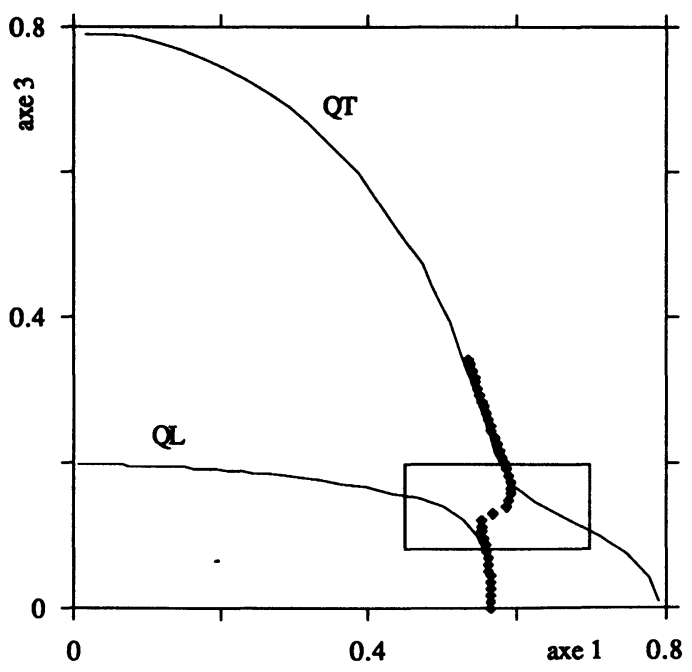

Fig. 3. - Valeurs expérimentales à $1 \mathrm{MHz}$ pour le même échantillon. Chevauchement de modes au voisinage du premier angle critique.

[Experimental points at $1 \mathrm{MHz}$ for the same sample. Overlapping of modes near the first critical angle.]

l'algorithme d'optimisation des constantes élastiques peut ne pas converger voire converger vers des valeurs farfelues. Un traitement de signal mieux adapté doit permettre la séparation des modes en présence d'une forte atténuation. 


\section{Utilisation de la transformation de Hilbert.}

La transformation de Hilbert qui est, au signe près, la convolution par $1 / \pi t$ possède la propriété intéressante de transformer un retard pur en une branche hyperbolique d'asymptote verticale plus facile à localiser que le maximum maximorum de l'intercorrélation entrée-sortie. En effet, reprenant la fonction de transfert de propagation sous la forme écrite en (5bis), sa transformée de Hilbert sera simplement :

$$
\operatorname{TH}(h)=g(t) *-1 / \pi(t-\tau) .
$$

Elle présente une branche hyperbolique d'asymptote verticale située en $t=\tau$ théoriquement indépendante du lissage par le filtre passe-bas $g(t)$. Une implantation sur micro-ordinateur a été réalisée utilisant des procédures classiques de transformation de Fourier rapide. Les propriétés bien connues de la transformation de Hilbert permettent d'écrire (10) sous la forme :

$$
\begin{array}{r}
\mathrm{TH}(h)=\mathrm{TF}^{-1}[H(f) \cdot i \cdot \operatorname{sign}(f)], \\
H(f)=S(f) / E(f)
\end{array}
$$

où $\mathrm{TF}^{-1}$ représente la transformée de Fourier inverse et $H(f)$ la fonction de transfert du matériau. Celle-ci est obtenue par division des transformées de Fourier du signal ayant transité à travers l'échantillon $S(f)$ et du signal de référence $E(f)$. Cette division est évidemment effectuée sur la bande passante maximum utilisable de $E(f)$.

Quelques exemples de signaux de synthèse permettent de voir l'influence sur le résultat, des conditions réelles liées à la quantification, l'échantillonnage et l'inévitable limitation pratique du domaine spectral.

\section{Simulations numériques.}

La simulation d'une impulsion ultrasonore est réalisée en modulant l'amplitude d'une sinusoïde de fréquence $f_{0}$ par une enveloppe de la forme

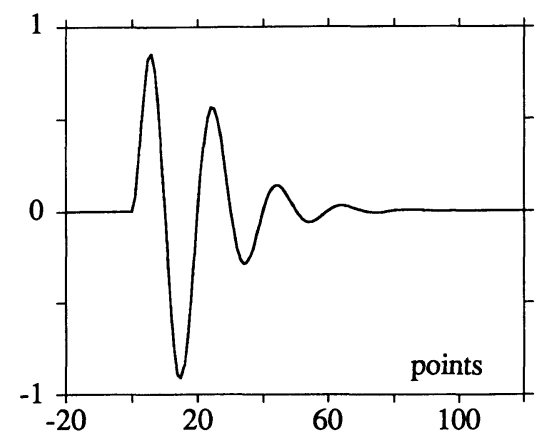

Fig. 4. - Signal synthétisé avec un retard nul sur 128 pas d'échantillonnage.

[Non delayed synthetic signal in a 128 sampling steps file.] $t$. $\exp (1-a t) . Y(t), Y(t)$ étant l'échelon de Heaviside. Ces signaux causaux représentent assez bien les formes expérimentales issues de transducteurs réels. Un exemple de simulation est représenté sur la figure 4 et son spectre sur la figure 5 .

L'étude d'un écho isolé se fait en prenant pour filtre un retard pur, soit $h(t)=\delta(t-\tau)$.

Les figures $6 \mathrm{a}$ et $6 \mathrm{~b}$ représentent les transformées de Hilbert obtenues par les relations (11). Les branches hyperboliques apparaissent modulant des oscillations. Celles-ci sont dues au calcul de $H(f)$ sur le domaine spectral de $E(f)$ limité à la bande $\pm N$, figure 5 .

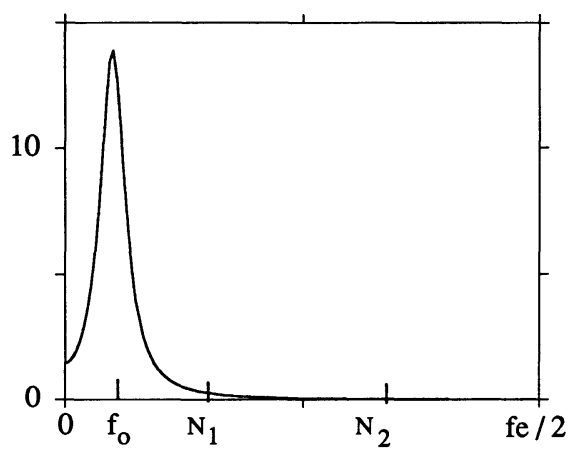

Fig. 5. - Spectre obtenu par transformée de Fourier rapide.

[Spectrum computed by Fast Fourier Transform.]

Avec un filtre centré sur l'origine et de largeur $2 \mathrm{~N}$ la transformée de Hilbert définie en (11) est remplacée par:

$\mathrm{TH}(h)=\delta(t-\tau) *(1 / \pi t) \cdot(\cos 2 \pi N t-1)$.

Les oscillations superposées à la branche hyperbolique sont d'autant plus serrées que la largeur du filtre est grande. La comparaison des figures $6 a$ et $6 \mathrm{~b}$ fait clairement apparaître que ces variations limitent la résolution de la méthode et que l'on aura donc intérêt à choisir la fréquence $N$ la plus grande possible compatible avec la dynamique spectrale des signaux tout en respectant la condition d'échantillonnage : $N<f_{\mathrm{e}} / 2$.

La lecture du retard s'effectue par localisation du passage à zéro entre deux branches hyperboliques. Une interpolation linéaire est effectuée entre deux échantillons successifs pour obtenir une incertitude inférieure au pas d'échantillonnage.

Pour illustrer l'influence d'un filtrage passe-bas sur la mesure du retard prenons dans (10), invariante par translation dans le temps, un retard nul et un filtre sans distorsion de phase du type $|G(f)|=\exp [-2 \pi a|f|]$ qui représente assez 


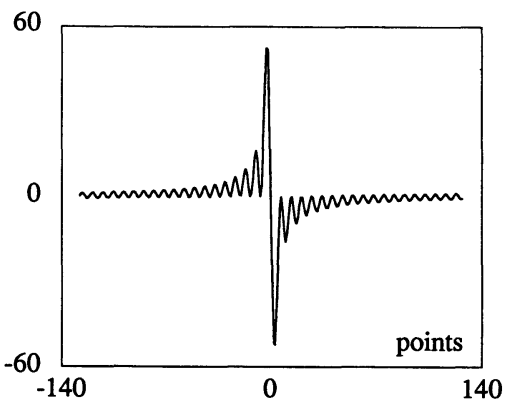

a)

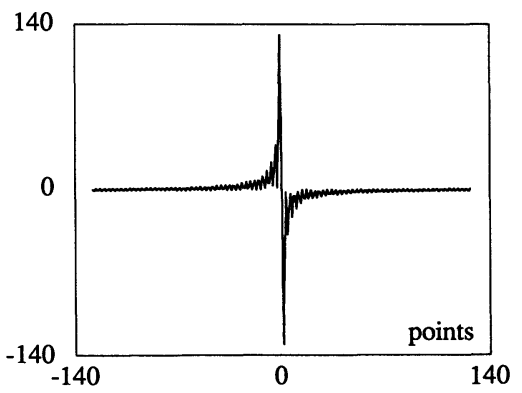

b)

Fig. 6. - (a) Filtrage serré $N_{1}=3 f_{0}$. (b) Filtrage large $N_{2}=6 f_{0}$.

[(a) Narrow band pass filtering $N_{1}=3 f_{0}$. (b) Wide band pass filtering $N_{2}=6 f_{0}$.]

bien les amortissements dans les polymères et pour lequel la transformée de Hilbert est connue :

$$
\mathrm{TH}(h)=-t / \pi\left(a^{2}+t^{2}\right) .
$$

La figure 7 montre que l'intersection avec l'axe des temps qui donne la valeur du retard est indépendante de la valeur $a$ de l'amortissement. Il n'en est pas de même, figure 8 , pour le maximum de l'inter-

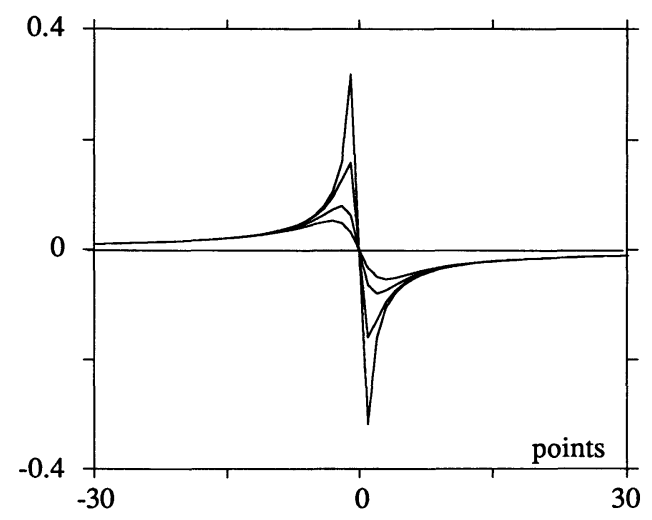

Fig. 7. - Evolution de l'enveloppe de la TH en fonction du filtrage.

[Hilbert transform shape versus low pass filtering.]

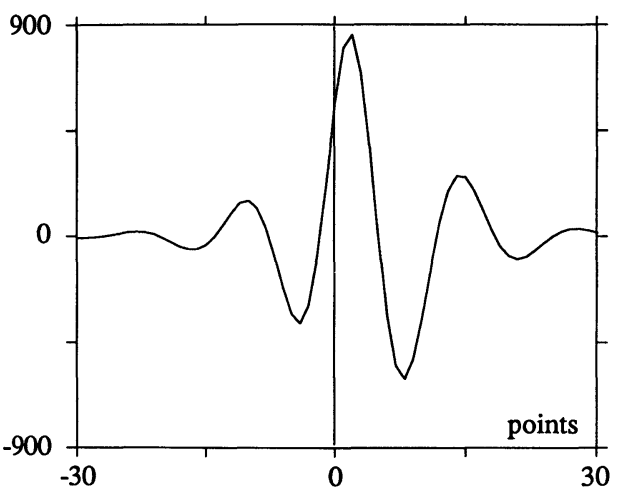

Fig. 8. - Décalage du maximum de l'intercorrélation par filtrage.

[Intercorrelation maximum shifted by low pass filtering.]

corrélation selon (9) décalée par ce filtrage passebas.

L'influence du chevauchement peut s'illustrer en considérant le signal composite somme de deux impulsions du type de la figure 5a d'amplitudes $A_{1}$ et $A_{2}$ et de retards $\tau_{1}$ et $\tau_{2}$. La réponse impulsionnelle est donc:

$$
h(t)=A_{1} \delta\left(t-\tau_{1}\right)+A_{2} \delta\left(t-\tau_{2}\right) .
$$

L'erreur $\varepsilon$ apportée dans la détermination de $\tau_{1}$ en fonction du décalage $\Delta \tau=\tau_{1}-\tau_{2}$, du rapport des amplitudes $A=A_{1} / A_{2}$ et pour une largeur de filtre $N$, s'exprime au premier ordre par :

$$
\varepsilon=-\left(A / 2 \pi^{2} N^{2}\right)(\cos 2 \pi N \Delta \tau-1) / \Delta \tau .
$$

Cette erreur est représentée sur la figure 9 pour un rapport d'amplitude de $+2 \mathrm{~dB}$ afin de comparai-

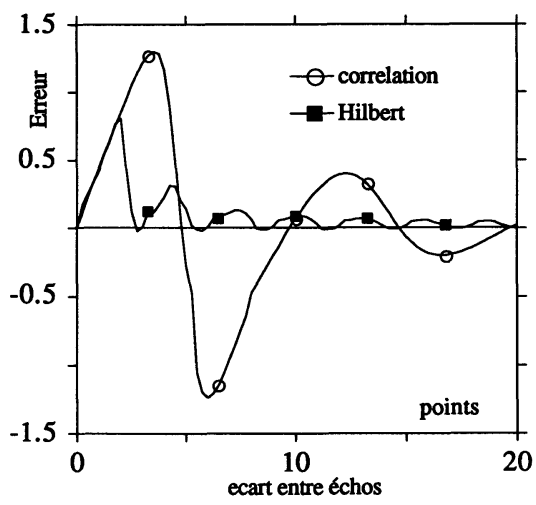

Fig. 9. - Erreur sur la mesure de retard du premier écho en présence d'un deuxième d'amplitude légèrement supérieure. Comparaison entre corrélation [6] et transformée de Hilbert.

[Error on the delay measurement for the first signal when a second one overlaps. Comparison between intercorrelation [6] and Hilbert transform.] 
son avec les mêmes valeurs obtenues par intercorrélation dans la référence [6].

Cette erreur variant comme $1 / N^{2}$, la précision sera d'autant meilleure que la division des spectres s'effectue sur une plus large bande. Toutefois, la localisation de deux zéros successifs dans la transformée de Hilbert n'est possible que s'il existe au moins deux points échantillonnés entre les deux branches pseudo-hyperboliques et si la période $1 / N$ des oscillations résultant de la limitation de bande est inférieure à l'écart entre les deux zéros.

Ces deux inéquations $\Delta \tau \geqslant 3 / f_{\mathrm{e}} \geqslant 1 / N$ sont compatibles avec le théorème d'échantillonnage qui requiert $2 N \leqslant f_{\mathrm{e}}$. Les figures 10 illustrent la détection de deux échos séparés de trois points, figure $10 \mathrm{~b}$, avec une fréquence d'échantillonnage égale à 20 fois la fréquence centrale $f_{0}$. La limite de séparation est de l'ordre du septième de période alors que la présence de deux échos superposés n'est pas perceptible à l'œil sur le signal temporel, figure 10a.

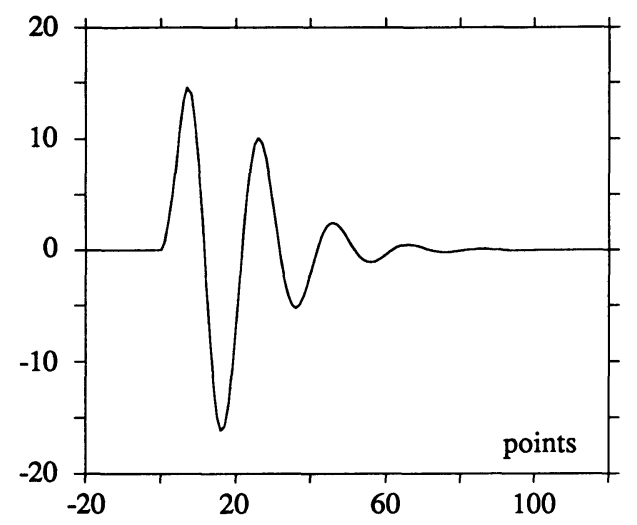

a)

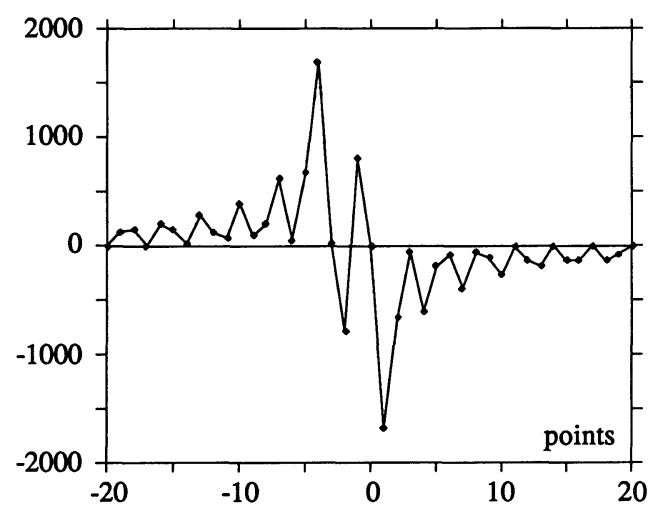

b)

Fig. 10. - (a) Somme de deux signaux identiques séparés de trois points. (b) Transformée de Hilbert avec $f_{\mathrm{e}}=20 f_{0}, N=6 f_{0}$.

[Overlapping of two same signals delayed by only three sampling steps. (b) Hilbert transform with $f_{\mathrm{e}}=20 f_{0}$, $N=6 f_{0}$.]

\section{Cas réels.}

La faible sensibilité à la dispersion de la méthode Hilbert est illustrée par une expérience de transmission au travers d'un échantillon en caoutchouc de silicone de $6 \mathrm{~mm}$ d'épaisseur. Sa fonction de transfert, figure 11, est obtenue par division des spectres entrée-sortie corrigée des coefficients de transmission interfaciaux dans la bande 1 à $7 \mathrm{MHz}$.

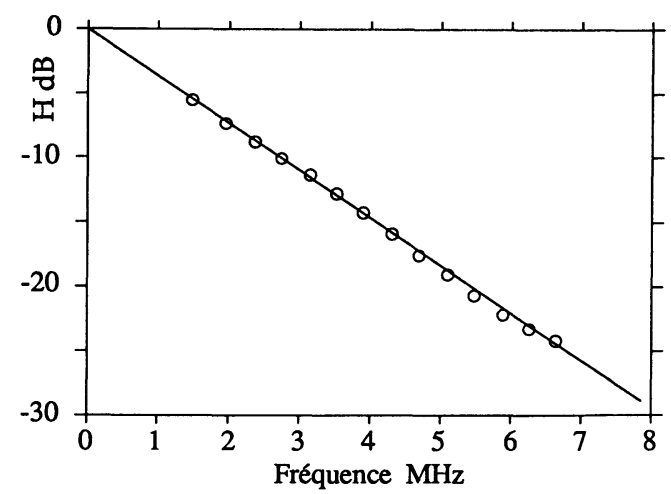

Fig. 11. - Fonction de transfert d'un échantillon en caoutchouc de silicone. Epaisseur : $6 \mathrm{~mm}$.

[Transfer function of a silicon rubber sample. Thickness : $6 \mathrm{~mm}$.]

On y relève une atténuation longitudinale importante proportionnelle à la fréquence vérifiant bien la relation (12) avec un coefficient $a=$ $625 \mathrm{~dB} / \mathrm{m}$. MHz. Les signaux acquis avec des transducteurs de fréquence centrale 0,5 et $5 \mathrm{MHz}$ ont été traités par trois méthodes différentes : seuillage à 10 niveaux de quantification, intercorrélation entréesortie et transformée de Hilbert. Les valeurs des retards obtenues, tableau suivant, montrent que la méthode Hilbert est la moins sensible à la dispersion.

\begin{tabular}{|c|c|c|}
\hline $\begin{array}{c}\text { Fréquence } \\
\text { centrale } \\
(\mathrm{MHz})\end{array}$ & & $\begin{array}{c}\text { Retards } \\
(\mu S)\end{array}$ \\
\hline 0,5 & 0,86 & 0,84 \\
\hline 5 & $\begin{array}{l}0,97 \\
\text { Seuil }\end{array}$ & $\begin{array}{l}0,91 \\
\text { Corré- } \\
\text { lation }\end{array}$ \\
\hline
\end{tabular}

La séparation de modes superposés est également meilleure avec cette méthode. Si l'on reprend l'expérience de transmission dans l'échantillon de carboneépoxy de la figure 3, le traitement par Hilbert fournit à $1 \mathrm{MHz}$ des courbes de lenteur (Fig. 12) identiques à celles obtenues à $5 \mathrm{MHz}$ (Fig. 2); l'algorithme d'optimisation de la matrice élastique converge alors vers les vraies valeurs avec une excellente fiabilité malgré le chevauchement des signaux visible sur la figure 1 . 


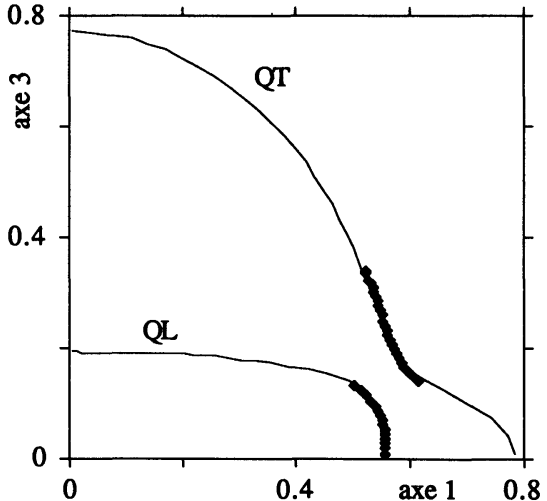

Fig. 12. - Lenteurs recalculées à $1 \mathrm{MHz}$ par transformée de Hilbert à comparer avec celles de la figure 3 obtenues par intercorrélation.

[Computed slownesses at $1 \mathrm{MHz}$ by Hilbert transform. Compare with intercorrelation figure 3.]

\section{Conclusion.}

Parmi les trois types de traitement les plus utilisés pour la mesure des temps de vol d'impulsions, la localisation des branches hyperboliques de la transformée de Hilbert apparaît à beaucoup de points de vue comme la mieux adaptée à ces problèmes d'évaluation non destructive.

$\mathrm{Si}$ l'on excepte le seuillage aux possibilités très limitées, les consommations de calcul sont identiques pour l'intercorrélation entrée-sortie et la transformée de Hilbert, l'une et l'autre utilisant les algorithmes de transformée de Fourier rapide. Par contre la division des spectres et la localisation des zéros sont plus exigeantes vis-à-vis de l'échantillonnage.

La meilleure résolution des échos chevauchants permet, dans les mêmes conditions expérimentales, de travailler à plus basse fréquence et donc de moins s'écarter des hypothèses de milieu homogène équivalent.

Enfin, le temps de vol mesuré par Hilbert est relié sans ambiguité à la seule partie réelle des constantes élastiques indépendamment de la nature et de la valeur de l'amortissement. En présence de dispersion, au contraire, l'intercorrélation entrée-sortie donne des valeurs que l'on ne sait relier ni à une vitesse de groupe ni à une vitesse de phase $[8,9]$ sans hypothèses supplémentaires sur les mécanismes d'atténuation. On découvre alors avec surprise des vitesses variant avec l'épaisseur de l'échantillon car la fréquence de coupure du filtre passe-bas équivalent lui est proportionnelle.

\section{Bibliographie}

[1] Markham M. F., Measurements of the elastic constants of fibre composites by ultrasonics, Composites 1 (1970) 45.

[2] Papadakis E. P., Ultrasonic velocity and Attenuation : Measurements methods with Scientific and Industrial Application, Phys. Acoust. XII (1976) 277-374.

[3] Roux J., Hosten B., Castagnede B., Deschamps M., Caractérisation mécanique des solides par spectro-interférométrie ultrasonore, Rev. Phys. Appl. 20 (1985) 351-358.

[4] Castagnede B., Thèse de spécialité $n^{\circ} 2001$, Université de Bordeaux I (1984).

[5] Hosten B., Castagnede B., Optimisation du calcul des constantes élastiques à partir des mesures de vitesses d'une onde ultrasonore, C. R. Hebdo. Acad. Sci. Paris II 296 (1983) 287-300.
[6] Castagnede B., Roux J. and Hosten B., Correlation method for normal mode tracking in anisotropic media using an ultrasonic immersion system. Ultrasonics 27 (1989) 280-287.

[7] Baste S., Deschamps M., Identification des constantes d'élasticité à partir des vitesses de propagation dans un plan principal de symétrie. C. $R$. Hebdo. Acad. Sci. Paris II 309 (1989) 1521-1526.

[8] Homes J.P., The experimental characterization of wave propagation systems ; II, Continuous systems and the effect of dispersion, J.S.V. 35 (1974) 52, 277-297.

[9] SACHSE W., PAO Yh., Determination of phase and group velocities of dispersive waves in solids, $J$. Appl. Phys. 49 (1978) 4320-4327. 